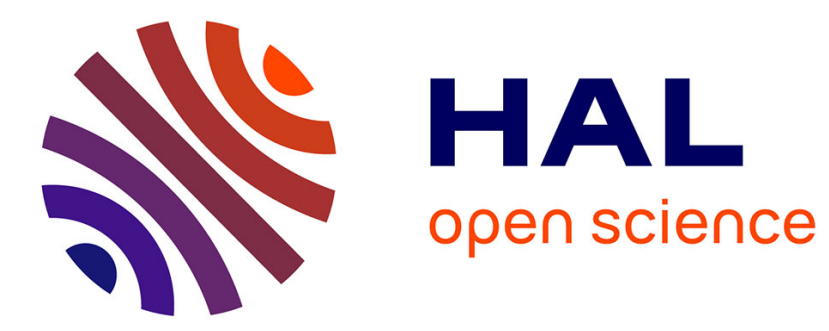

\title{
Airfoil Shape Optimization for Transonic Flows of Bethe-Zel'dovich-Thompson Fluids
}

Paolo Maria Congedo, Christophe Eric Corre, Paola Cinnella

\section{To cite this version:}

Paolo Maria Congedo, Christophe Eric Corre, Paola Cinnella. Airfoil Shape Optimization for Transonic Flows of Bethe-Zel'dovich-Thompson Fluids. AIAA Journal, 2007, pp.1303-1316. 10.2514/1.21615. hal-00787552

\section{HAL Id: hal-00787552 https://hal.science/hal-00787552}

Submitted on 15 Feb 2013

HAL is a multi-disciplinary open access archive for the deposit and dissemination of scientific research documents, whether they are published or not. The documents may come from teaching and research institutions in France or abroad, or from public or private research centers.
L'archive ouverte pluridisciplinaire HAL, est destinée au dépôt et à la diffusion de documents scientifiques de niveau recherche, publiés ou non, émanant des établissements d'enseignement et de recherche français ou étrangers, des laboratoires publics ou privés. 


\title{
Airfoil Shape Optimization for Transonic Flows of Bethe-Zel'dovich-Thompson Fluids
}

\author{
P. M. Congedo* \\ University of Lecce, 73100 Lecce, Italy \\ C. Corre \\ Ecole Nationale Supérieure d'Arts et Métiers, 75013 Paris, France \\ and \\ P. Cinnella \\ University of Lecce, 73100 Lecce, Italy
}

\begin{abstract}
High-performance airfoils for transonic flows of Bethe-Zel'dovich-Thompson fluids are constructed using a robust and efficient Euler flow solver coupled with a multi-objective genetic algorithm. Bethe-Zel'dovichThompson fluids are characterized by negative values of the fundamental derivative of gasdynamics for a range of temperatures and pressures in the vapor phase, which leads to nonclassical gasdynamic behaviors such as the disintegration of compression shocks. Using Bethe-Zel'dovich-Thompson gases as working fluids may result in low drag exerted on airfoils operating at high transonic speeds, due to a substantial increase in the airfoil critical Mach number. This advantage can be further improved by a proper design of the airfoil shape, also leading to the enlargement of the airfoil operation range within which Bethe-Zel'dovich-Thompson effects are significant. Such a result is of particular interest in view of the exploitation of Bethe-Zel'dovich-Thompson fluids for the development of high-efficiency turbomachinery.
\end{abstract}

\section{Introduction}

D ENSE gases (DGs) are defined as single-phase vapors operating at temperatures and pressures of the order of magnitude of those of their thermodynamic critical point. At these conditions, real gas effects play a crucial role in the gasdynamic behavior of the fluid. The study of the complicated dynamics of compressible flows of dense gases is strongly motivated by their potential technological advantages as working fluids in energyconversion cycles and, specifically, in organic Rankine cycles (ORCs).

Specific interest has developed in a particular class of dense gases, known as the Bethe-Zel'dovich-Thompson (BZT) fluids [1], which exhibit nonclassical gasdynamic behaviors in a range of thermodynamic conditions above the liquid/vapor coexistence curve, such that the fundamental derivative of gasdynamics

$$
\Gamma: 1+\frac{\rho}{a}\left(\frac{\partial a}{\partial \rho}\right)_{s}
$$

where $\rho$ is the fluid density, $a$ is the sound speed, and $s$ is the entropy, which becomes negative. At these conditions, the well-known compression shocks of the perfect-gas (PFG) theory violate the entropy inequality over a certain range of temperatures and pressures in the vapor phase and are therefore inadmissible [1-3]. The thermodynamic region characterized by negative values of $\Gamma$ is usually called the inversion zone, and the $\Gamma=0$ contour is called the transition curve. BZT properties are generally encountered in fluids possessing large heat capacities and formed by complex, heavy

*Ph.D. Student, Department of Engineering for Innovation, via Monteroni. Member AIAA.

${ }^{\dagger}$ Assistant Professor, SINUMEF Laboratory, 151 Boulevard de l'Hôpital.

¥Assistant Professor, Department of Engineering for Innovation, via Monteroni. Member AIAA. molecules, such as some commercially available heat transfer fluids. The nonclassical phenomena typical of BZT fluids have several practical outcomes: prominent among them is an active research effort to reduce losses caused by wave drag and shock/boundarylayer interactions in turbomachines and nozzles [4-7], with particular application to ORCs.

ORCs are used to generate electric energy in low-power applications. They work in the same way as classical steam Rankine cycles, but due to the use of low-boiling compounds as working fluids, they can use low-temperature heat sources such as geothermal and solar sources or waste heat from industrial applications. ORCs typically use a single expansion stage, which operates in the transonic/supersonic regime. One of the major loss mechanisms in transonic and supersonic turbomachinery is related to the generation of shock waves. Past research efforts toward demonstrating the existence of BZT fluids [8-10] indicate that some heavy compounds employed for heat transfer applications and as ORC working fluids possess BZT properties. Therefore, shock formation and the consequent losses could be ideally avoided if turbine expansion could happen entirely within or very close to the inversion zone. Previous works on BZT transonic flows past airfoils [5] and on BZT flows through turbine cascades [6,7] generally considered operation conditions in the very neighborhood of the $\Gamma=0$ curve, just above the upper saturation curve. In such conditions, the flowfield evolves almost entirely within the inversion zone and no compressive shock waves are formed, leading to an almost isentropic expansion through the entire cascade. Unfortunately, the inversion zone has a quite limited extent; thus, a reduction in the temperature jump between the heater and condenser stages is generally required to completely operate the turbine cascade in the BZT regime. As a consequence, the risk is to enhance turbine efficiency at the cost of a lower global cycle power output and overall cycle efficiency. This important drawback has been the stumbling block to the development of real-world BZT organic Rankine cycles.

Recently, an alternative approach was explored by two of the present authors [11]. To enlarge the system operating range, the flow is allowed to evolve, in part, outside the inversion zone. With this choice, compression shocks and mixed waves can occur, causing losses; however, such waves are expected to be relatively weak if they have jump conditions in the vicinity of the transition curve [2]. 


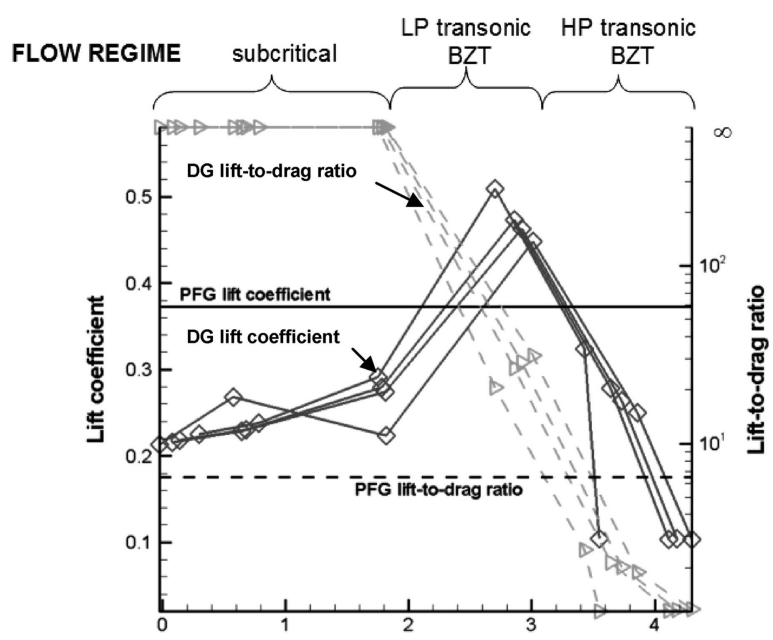

Freestream value of the gas dynamics fundamental derivative

a)

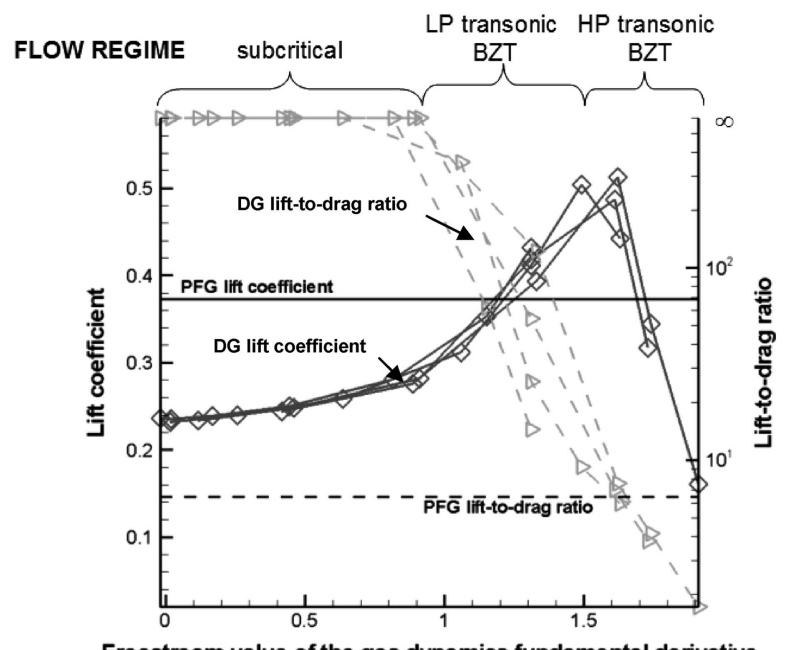

Freestream value of the gas dynamics fundamental derivative

b)

Fig. 1 Typical evolution of the NACA0012 aerodynamic performances at $M_{\infty}=0.85$ and $\alpha=1 \mathrm{deg}$ for a dense-gas flow: a) results for a densegas flow of BZT VDW gas, taken from [11] and b) results for PP10 fluorocarbon under the MAH thermodynamic model, taken from [12]; perfect-gas results are also shown for comparison.

This means that the associated losses will be lower than normal, leading to high turbine efficiency; in turn, operating the turbine cascade partially outside the inversion zone allows an increase in the temperature jump of the power cycle. The feasibility of such an approach was studied for a simplified configuration, represented by an isolated NACA0012 airfoil in the transonic regime, using the van der Waals (VDW) equation of state for polytropic gases, which is the simplest thermodynamic model accounting for BZT effects. Figure 1a summarizes the results obtained in [11] when computing the inviscid flow of a polytropic BZT van der Waals gas at Mach 0.85 and 1-deg angle of attack over the NACA0012 airfoil for various freestream thermodynamic conditions, corresponding to a series of operation points selected along isentropes crossing the inversion zone. For each operation point, the lift coefficient and lift-to-drag ratio are plotted as a function of the freestream value taken by the fundamental derivative of gasdynamics $\Gamma_{\infty}$. Please note that the fundamental derivative does not vary monotonically along an isentrope: it decreases when approaching the inversion zone from the low-pressure side, reaches a minimum within it, and then increases again. Nevertheless, as the operating region of interest for ORCs is located at the high-pressure side of the inversion zone, only the lefthand branch of the isentrope is considered, such that $\Gamma_{\infty}$ monotonically increases with increasing pressure. The parametric study allowed us to identify three flow regimes depending on the value of $\Gamma_{\infty}$. For small values of $\Gamma_{\infty}$ in the subcritical regime, the liftto-drag ratio tends to infinity (for inviscid flows), because the flow remains subsonic everywhere, thus avoiding the occurrence of wave drag. When $\Gamma_{\infty}$ is in the range of $2-3$, a significant growth in both lift and drag is observed. The increase in lift is produced by the formation of an expansion shock close to the leading edge that strongly enhances the suction peak at the airfoil upper surface. The increase in drag is due to the occurrence of shocks on the airfoil surface. In this regime, called the low-pressure (LP) transonic BZT regime, the liftto-drag ratio remains one order of magnitude greater than in the PFG case. Finally, when $\Gamma_{\infty}$ reaches higher values in the so-called highpressure (HP) transonic BZT regime, the flow becomes qualitatively similar to that of a PFG, with even poorer aerodynamic performances. Similar qualitative conclusions were drawn in $[12,13]$, in which the parametric study was repeated using the more realistic Martin-Hou (MAH) equation of state (EOS). As indicated in Fig. $1 \mathrm{~b}$, the difference introduced by the change of EOS lies in the extent of the intermediate LP transonic BZT regime, now taking place for values of $\Gamma_{\infty}$ in the range of $1-1.5$. Viscous effects taken into account in [13-15] do not alter this qualitative behavior. An important conclusion that can be drawn from the studies performed in [11-15] is that the choice of upstream conditions within or very close to the $\Gamma=0$ line is not optimal in terms of aerodynamic performance. A better choice is to work in the LP transonic BZT regime, which offers the best compromise between high lift and low drag.

Note, however, that these results were obtained for a specific airfoil; it may be possible to find an airfoil with the same thickness-tochord ratio that offers higher levels of lift in the subcritical regime (in particular, higher than the level obtained for a PFG flow), while preserving lift-to-drag ratios in the LP transonic BZT flow regime (in which the BZT effects are less strong) that are one order of magnitude larger than the ratio associated with PFG flow. In practice, it is specially important to ensure high aerodynamic performances in both regimes, because the freestream pressure is likely to change according to the turbine operating conditions.

The present paper is devoted to finding such optimal airfoil geometries for transonic DG flows by making use of evolutionary optimization strategies, namely, multi-objective genetic algorithms (MOGAs). Shape optimization for DG flows has rarely been treated in the past. To the authors' best knowledge, the only previous works on the subject are provided by $[16,17]$, which consider the construction of low-drag shapes for nonlifting inviscid flows of DGs through the transonic small-disturbance theory. In [16], similarity solutions of a nonlinear small-disturbance equation are studied, describing a two-dimensional near-sonic potential flow of DGs. The solutions are applied to the problem of a near-sonic smalldisturbance flow of DGs in the surrounding of two-dimensional, slender, semi-infinite bodies with $x^{n}$ generators, in which $2 / 7<$ $n<1$ and $x$ is the distance along the body axis. In the case when $n=2 / 7$, the body constitutes a surface over which the flow is sonic at every point and the pressure distribution is constant. The analysis indicates that such a shape is optimal for near-sonic flows of DGs, in the sense that it offers the minimal pressure drag. In [17], low-drag airfoils are constructed through a nonlinear small-disturbance theory. These airfoils exhibit a higher critical Mach number (i.e., the freestream Mach number at which sonic flow first appears on the airfoil) with respect to nonoptimized shapes. Consequently, they yield zero wave drag over a larger range of incoming flow velocities. Specifically, the analytical study presented in [17] derives two airfoil geometries with a $12 \%$ thickness-to-chord ratio that show lower wave drag than the NACA0012 airfoil at zero incidence for specific freestream thermodynamic conditions $\left(\Gamma_{\infty}=0\right)$. The first airfoil geometry is formed by an arc along which the flow is always sonic; the second one is a modified airfoil obtained by smoothly connecting the head of the previous sonic arc with a sharp tail, to prevent boundary-layer separation for viscous flows. Reference [17] has the merit of facing, for the first time, optimization problems for DG flows past airfoils, even though an optimization procedure in the proper sense is not undertaken. In practice, such an approach is incomplete, because it does not take into account the effect of the optimization on 
the airfoil lift. Moreover, the small-disturbance approximation does not accurately model the important flow variations at the airfoil leading and trailing edges, which have a crucial influence on the airfoil performance, especially for high- $\Gamma$ incoming-flow conditions.

In the present work, we consider two optimization problems: the first one, as in Rusak and Wang's work [17], aims at finding a minimal-drag airfoil shape for a nonlifting flow in the BZT regime. The flowfield is computed by solving the complete Euler equations, thus fully taking into account all of the nonlinear effects. This symmetric problem is relatively simple to treat, because the flow symmetry reduces the computational expense, whereas a single objective function to minimize (namely, the drag) implies lower optimization complexity. It will be shown that if the optimal airfoil shape derived in [17] does exhibit lower drag than a reference airfoil with the same thickness-to-chord ratio (viz, the NACA0012 airfoil), the present approach allows us to obtain even higher performances by combining an efficient Euler solver to a genetic algorithm (GA). The second application deals with the optimization of a lifting airfoil. The following optimization strategy is adopted: find an airfoil shape that allows us to obtain high lift at BZT subcritical conditions (in which the wave drag is in any way expected to be zero) and to minimize wave drag while maximizing lift for supercritical BZT flow conditions.

The paper is organized as follows: in Sec. II, some of the peculiar properties of DG flows are briefly recalled and the thermodynamic models used for the present study are presented, with some comments about their main advantages and drawbacks; Sec. III is devoted to the description and validation of the flow solver and provides details of the present optimization strategy; finally, optimization results are discussed in Sec. IV for both the symmetric airfoil drag reduction problem and for the lifting-airfoil problem, and comparisons are made with the optimized results obtained for a PFG flowing at the same conditions.

\section{A. Governing Equations}

\section{Dense-Gas Modeling}

Dense-gas flows are governed by the equations for equilibrium, nonreacting flows. In the present study, we consider the Euler equations, written in integral form for a control volume $\Omega$ with boundary $\partial \Omega$ :

$$
\frac{d}{\mathrm{~d} t} \int_{\Omega} w \mathrm{~d} \Omega+\int_{\partial \Omega} \boldsymbol{f} \cdot \boldsymbol{n} \mathrm{d} S=0
$$

In Eq. (2), $w$ is the conservative variable vector, where

$$
\boldsymbol{w}=(\rho, \rho \boldsymbol{v}, \rho E)^{T}
$$

$\boldsymbol{n}$ is the outer normal to $\partial \Omega$ and $\boldsymbol{f}$ is the flux density:

$$
\boldsymbol{f}=(\rho v, p \overline{\bar{I}}+\rho \boldsymbol{v} \boldsymbol{v}, \rho \boldsymbol{v} H)^{T}
$$

where $\boldsymbol{v}$ is the velocity vector, $E$ is the specific total energy, $H=$ $E+p / \rho$ is the specific total enthalpy, $p$ is the pressure, and $\overline{\bar{I}}$ is the unit tensor. The preceding equations are completed by a thermal and a caloric equation of state:

$$
\begin{aligned}
& p=p[\rho(\boldsymbol{w}), T(\boldsymbol{w})] \\
& e=e[\rho(\boldsymbol{w}), T(\boldsymbol{w})]
\end{aligned}
$$

where $e$ is the specific internal energy and $T$ the absolute temperature.

\section{B. Dense-Gas Properties}

The dynamic behavior of dense gases is governed by the fundamental derivative of gasdynamics (1) which, using standard thermodynamic manipulations, can be rewritten as follows:

$$
\Gamma=\frac{v^{3}}{2 a^{2}}\left(\frac{\partial^{2} p}{\partial v^{2}}\right)
$$

where $v=1 / \rho$ is the fluid specific volume, and $\Gamma$ represents a measure of the rate of change of the sound speed $a=\left[-v^{2}(\partial p / \partial v)_{s}\right]^{\frac{1}{2}}$. If $\Gamma<1$, the flow exhibits an uncommon sound-speed variation in isentropic perturbations: $a$ grows in isentropic expansions and drops in isentropic compressions, contrary to what happens in "common" fluids. For heavy gases, composed of sufficiently complex molecules and characterized by high $c_{v} / R$ ratios (where $c_{v}$ is the constant volume specific heat and $R$ is the gas constant), $\Gamma$ is smaller than one, or even smaller than zero, for extended ranges of densities and pressures and recovers its perfectgas value in the low-density limit. The sign of $\Gamma$ is univocally determined by the sign of the second derivative $\left(\partial^{2} p / \partial v^{2}\right)_{s}$, that is, the concavity of the isentrope lines in the $p-v$ plane. It is possible to show [2] that the entropy change across a weak shock can be written as

$$
\Delta s=-\frac{a^{2} \Gamma}{v^{3}} \frac{(\Delta v)^{3}}{6 T}+\mathcal{O}\left[(\Delta v)^{4}\right]
$$

where $\Delta$ represents a change in a given fluid property through the shock. As a result, if $\Gamma>0$, a negative change in the specific volume (i.e., a compression) is required to satisfy the second law of thermodynamics, whereas a positive change (i.e., an expansion) is the only physically admissible solution when $\Gamma<0$. BZT fluids are precisely defined as fluids that exhibit a region of negative $\Gamma$ in the vapor phase. An important property of BZT fluids is that the shock strength is reduced up to one order of magnitude from that predicted by Eq. (6) for thermodynamic conditions for which $\Gamma \approx 0$. Cramer and Kluwick [2] showed, in fact, that $\Gamma=\mathcal{O}(\Delta v)$ for small volume changes in the vicinity of the transition curve. Thus, shock waves having jump conditions in the thermodynamic region near the $\Gamma=0$ contour are expected to be much weaker than normal.

\section{Thermodynamic Models}

In the present work, two gas models are considered. The VDW equation of state is the earliest attempt to correct the perfect-gas law to take into account covolume effects and attractive intermolecular forces; it only satisfies two thermodynamic constraints: the horizontal slope and the inflection of the critical isotherm at the critical point. However, these are sufficient conditions to allow the VDW equation to model BZT fluid behavior. In fact, in the limit $c_{v} / R \rightarrow \infty$, the isentropes and isotherms coincide. Therefore, a van der Waals gas with sufficiently high $c_{v} / R$ ratio is expected to exhibit reversed isentrope concavity above the upper saturation curve; hence, it possesses BZT properties. It is possible to show [5] that by taking the specific heat ratio $\gamma$ in the range of $1<\gamma<1.06$, a region of negative values of the fundamental derivative appears. The VDW thermodynamic model is computationally inexpensive and has been often used to provide a qualitative description of BZT fluid flows [4,16-20]. However, it is not very accurate, and it has been proven [9] that it largely overestimates the extent of the inversion zone. Nevertheless, if this inaccuracy affects quantitative results obtained for a given gas, it does not affect the qualitative behavior significantly; the results obtained are roughly representative of the thermodynamic response of a real-world BZT gas with higher specific heats.

The second gas model considered here is the comprehensive equation of state of Martin and Hou [21], involving five virial terms and satisfying ten thermodynamic constraints. This equation is one of the best available gas models to manageably compute dense-gas effects $[22,23]$ and provides an accurate description of the gas behavior close to the saturation curve by using just a few input thermodynamic data. However, it is considered to somewhat underestimate the extent of the inversion zone; for this reason, results provided by the MAH model are likely to be conservative.

Figure 2 shows the $p-v$ diagram and the inversion zone for a polytropic VDW gas, computed using an input value for the specific 


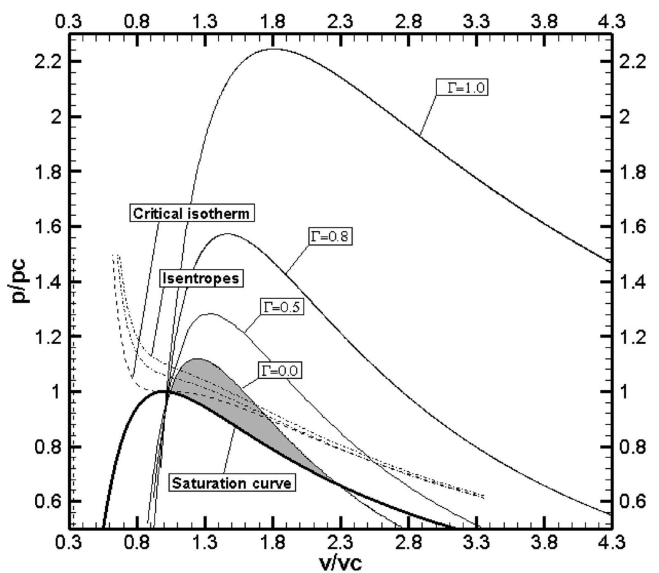

a)

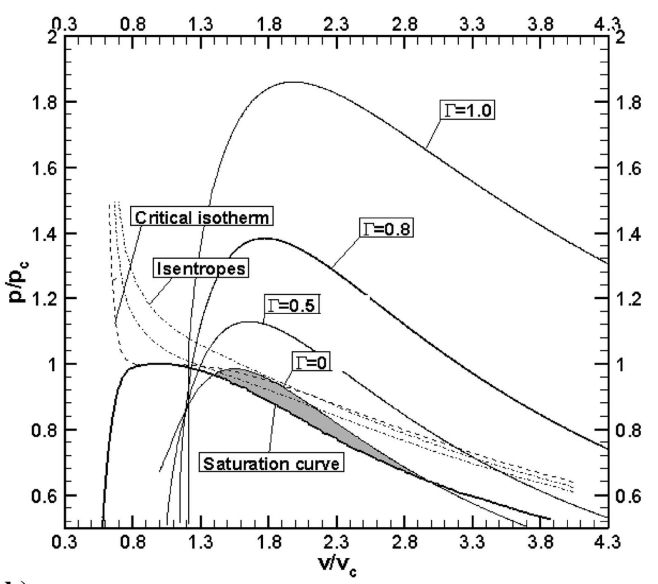

b)

Fig. 2 Amagat diagrams for a) VDW BZT gas with $\gamma=1.0125$ and b) heavy fluorocarbon PP10 modeled through the MAH EOS; the shaded region represents the inversion zone.

heat ratio corresponding to the limiting perfect-gas value for heavy fluorocarbons $(\gamma \approx 1.0125)$ and for the heavy fluorocarbon PP10, under the MAH thermodynamic model. Note the different shape of the saturation curve and the different shape and size of the inversion zone obtained with the two gas models.

\section{Flow Solvers and Genetic-Based Optimization Tool}

\section{A. Flow Solvers}

Two DG flow solvers are used in the present study: a newly developed unstructured-grid solver (UGS) is retained for the geneticbased optimization stage (for reasons detailed later), and an existing structured-grid solver (SGS) used in previous DG studies [11-15] is applied for cross-validation purposes and a posteriori analysis of optimal geometries. For the SGS, the governing equations are discretized using a cell-centered finite volume scheme of third-order accuracy, which allows computing flows governed by an arbitrary equation of state [15]. The scheme is constructed by correcting the dispersive error term of the second-order-accurate Jameson's scheme [24]. The use of a scalar dissipation term simplifies the scheme implementation with highly complex equations of state and greatly reduces computational costs. To preserve the high accuracy of the scheme on non-Cartesian grids, the numerical fluxes are evaluated using weighted discretization formulas, which take into account the stretching and the skewness of the mesh; this ensures true third-order accuracy on moderately deformed meshes and at least second-order accuracy on highly distorted meshes (see [25] for details). The equations are then integrated in time using a four-stage Runge-Kutta scheme [24]. Local time-stepping, implicit residual smoothing, and a multigrid are used to efficiently drive the solution to the steady state.
For external flows, nonreflecting boundary conditions based on a multidimensional method of characteristics are applied at far-field boundaries; an adiabatic wall condition is imposed at solid boundaries.

The UGS is also based on a cell-centered finite volume discretization of Eq. (2), but formulated on a general unstructured grid dividing the spatial domain into a finite number of triangles or quadrangles. The time rate of change of the cell-averaged state vector $\boldsymbol{w}$ is balanced with the area-averaged (inviscid) fluxes across the cell faces. The fluxes are computed across each cell face using the HLL scheme [26]. Second-order spatial accuracy is ensured by means of a MUSCL-type reconstruction process on the conserved variables [27], in which the gradient estimates required at each cell center are obtained through a least-squares formula. Because the solver will be applied to the computation of flows containing discontinuities, the reconstruction formula includes a limitation step based on the now standard approach proposed in [28] and revisited in [29], which ensures oscillation-free shock-capturing. Fast convergence to steady state is provided by making the scheme implicit, following a procedure inspired from [30], in which a simple first-order Rusanovtype implicit stage allows the use of large CFL numbers and is solved by an inexpensive point-relaxation technique. The numerical flux through the boundary edges is computed using an inflow/outflow characteristic-based condition to define the ghost-cell states at the far-field boundary and a mirror boundary condition to define the ghost-cell states at the wall.

With the accuracy properties of the SGS used in this work having been previously demonstrated $[11,23]$, the validation study will focus on the results provided by the UGS, which will be compared with the reference results obtained from the SGS. As a first validation test, both solvers are applied to the computation of inviscid flows over a NACA0012 airfoil at zero angle of attack for different values of the Mach number. A series of calculations is run with the UGS for a half-profile on a grid made of 6400 quadrilateral cells with a grid size of $5 \times 10^{-3} c$ at the wall, where $c$ denotes the airfoil chord, 120 points along the airfoil surface, and an outer boundary about 10 chords away from the airfoil. The analysis provided in [11] showed that such a flow is accurately computed using the SGS in a $136 \times 40$ grid with a mean height of the first cell closest to the wall of about $5 \times 10^{-3} c$ and outer boundary located 20 chords away. The evolution of the drag coefficient is computed with both solvers in the case of a PFG flow $(\gamma=1.4)$ and in the case of a DG flow. In the latter case, following [17], the freestream pressure and specific volume normalized by their critical values are taken equal to $p_{\infty} / p_{c}=$ 1.0696 and $\rho_{\infty} / \rho_{c}=0.73502$, and the working fluid is a VDW gas with $\gamma=1.02$. As is well known from previous studies $[4,5,11,17,20,31]$, the use of a BZT gas allows a tremendous increase of the critical Mach number. From the present computations, it is found that the flow becomes sonic at the wall



Fig. 3 Drag coefficient of the NACA0012 airfoil at zero incidence as a function of Mach number for a perfect and dense-gas flow; UGS and SGS results. 
for a freestream Mach number of about 0.83 for the PFG flow and above 0.90 for the DG flow. Consequently, the drag divergence resulting from the occurrence of shock waves on the airfoil surface is much delayed when using a BZT gas instead of a perfect diatomic gas, as shown in Fig. 3. The drag coefficient prediction provided by the UGS for both PFG and DG flow remains close to that of the SGS for the whole range of freestream Mach number. For the DG case, numerical results from [17] are also reported for further comparison and validation, showing good agreement with the present results. The possibility of further increasing the critical Mach number, further reducing the drag, through a proper shaping of the airfoil will be investigated in the next section.

A second validation of the UGS is provided by the computation of the DG flow over the same NACA0012 airfoil at $M_{\infty}=0.85$ and $\alpha=1 \mathrm{deg}$; the dense gas is modeled using the MAH equation of state with coefficients corresponding to PP10. Two operation points of interest are selected: the freestream thermodynamic conditions $p_{\infty} / p_{c}=1.008, \rho_{\infty} / \rho_{c}=0.676$, and $\Gamma_{\infty}=0.168$, denoted hereafter as OP 1, yield significant BZT effects, whereas the choice of $p_{\infty} / p_{c}=1.079, \rho_{\infty} / \rho_{c}=0.882$, and $\Gamma_{\infty}=1.312$, denoted as OP 2 from now on, leads to higher values of the fundamental derivative throughout the flow and, thus, to much reduced BZT effects. Numerical solutions corresponding to these operation points are computed using the SGS on a $256 \times 44 \mathrm{O}$-grid and the UGS on a grid made of 14,374 triangular elements. In both cases, the mean height of the closest cell to the wall is about $5 \times 10^{-3} c$, and the far-field boundary is located 10 chords away from the airfoil. Here again, the results obtained with the SGS scheme in [11] are considered as the reference to which the UGS scheme results will be compared. The wall pressure and Mach number distributions computed by both solvers are displayed in Fig. 4 , along with PFG flow $(\gamma=1.4)$


Fig. 4 Wall distributions of the pressure coefficient and Mach number for perfect and dense-gas flows over a NACA0012 airfoil at $M_{\infty}=0.85$ and $\alpha=1 \mathrm{deg}$. distributions; the UGS results are in excellent agreement with the SGS ones. The DG flow for OP 1 remains subcritical with a computed lift coefficient $C_{L}=0.2265$ for the SGS and $C_{L}=0.2244$ for the UGS and a drag coefficient very close to zero. For OP 2, the DG flow becomes supercritical with $C_{L}=0.4467$ and $C_{D}=0.0510$ for the SGS and with $C_{L}=0.4489$ and $C_{D}=0.0496$ for the UGS. The difference between the SGS and UGS on lift prediction therefore remains within $1 \%$ and that on drag prediction does not exceed $3 \%$. Note the PFG flow is such that $C_{L}=0.373$ and $C_{D}=0.0574$; thus, as already explained in the Introduction, though the dense gas allows achieving an extremely high lift-to-drag ratio in the subcritical OP 1 conditions, the corresponding lift level remains below that of the PFG flow, whereas the DG aerodynamic performance in the supercritical OP 2 conditions tends to come close to that of the perfect gas. The possibility to simultaneously achieve higher lift values for operating conditions in which BZT effects are important and to improve the lift-to-drag ratio when these effects become less significant is addressed in the next section.

\section{B. Pareto-Based Genetic Algorithm}

Both the reduction of drag for a nonlifting airfoil and the multipoint performance improvement of a lifting airfoil in a densegas stream can be expressed as shape-optimization problems. These problems are solved in an automatic way by coupling a DG flow solver and a mesh generator with an optimizer. The flow solver used in the optimization process is the UGS: its accuracy properties have proved satisfactory when compared with the SGS, it offers fast convergence to steady state (due to its implicit formulation), and it can be readily coupled with an unstructured grid generator. As for the optimization tool, it was decided to make use of a MOGA. Genetic algorithms were successfully applied for some time now to shape optimization in aeronautics [32-35]. In spite of their cost, GAs have proved their usefulness with respect to gradient-based methods, because of their high flexibility [stemming from the fact that they only require values of the objective function(s) to efficiently explore the parameter space in search of an optimum] and also because of their ability to find global optima of multimodal problems.

Moreover, so-called Pareto-type genetic algorithms are of particular interest for multi-objective optimization, because they provide a set of nondominated solutions after only a few generations. On the contrary, a conventional gradient-based method needs several independent runs to achieve similar results $[35,36]$. In the present work, the flexibility offered by the genetic algorithm will allow an immediate switch from VDW to MAH equations of state and leaves the door open for the future use of the SGS as the flow solver; the MOGA is also well suited to the solution of multi-objective problems such as multipoint performance improvement for a lifting airfoil. The MOGA applied in this study is the nondominated sorting genetic algorithm proposed by Srinivas and Deb [37]. At a given generation number, all individuals in the population are ranked according to nondomination criteria that allow taking into account a set of objective functions in a simultaneous way.

The set of individuals that dominate all of the other members of the population without dominating each other is designated as the front of rank 1, the set of dominant individuals in the population deprived from the members of rank 1 form the front of rank 2, and so forth, until the whole population is classified into a series of dominance fronts. The individuals scattered along the front of rank 1 are assigned the same pseudofitness function, arbitrarily fixed to unity, because they are equally well-adapted and therefore should be given the same potential of reproduction. However, to favor the population diversity along the front, the pseudofitness value is decreased for individuals located in crowded areas of the front: practically, the initial uniform value of the pseudofitness function is divided by a number strictly larger than unity for individuals with neighbors within a prescribed distance. Next, the smallest value of the modified pseudofitness function obtained for individuals belonging to the front of rank 1 is decreased from a small number $\varepsilon$ and assigned to all members of the front of rank 2; this uniform value is next itself modified, as previously explained, to promote the selection of 




Fig. 5 Evolution of the maximal and mean value of the drag coefficient for the computed population as a function of the number of generations; mono-objective (single operation point) optimization runs for different values of the freestream Mach number.

isolated individuals along the front and thus to ensure a maximum of diversity. Once each individual has been given a pseudofitness value, it is selected for reproduction using a proportional selection operator; finally, the individuals retained in the mating pool are submitted to crossover and mutation, to explore the research space and yield a front of rank 1 increasingly close to the global Pareto front of the problem.

The main tuning parameters of the algorithm are the population size, the number of generations, the crossover and mutation probabilities $p_{c}$ and $p_{m}$, and the so-called sharing parameter $\sigma$ (used to take into account the relative isolation of an individual along a dominance front). Typical values for $p_{c}$ and $p_{m}$ are, respectively, 0.9 and 0.1 ; values of $\sigma$ are retained following a formula given in [35], which takes into account the population size and the number of objectives. Theoretically, the population size and the number of generations should be chosen according to the number of parameters and objectives of the optimization problem under study; in practice, however, the population size and number of generations are fixed by the global amount of CPU time devoted to the computation: taking too small of a population may rapidly lead to a local optimum and make useless the iteration process on the generation number; conversely, a large population will impose a limited number of generations, which could not allow the population to evolve sufficiently toward the optimum. Because each inviscid flow computation performed in the present study takes between 5 and 10 min on a PC equipped with a Pentium Pro processor, requesting a whole optimization run to be completed in less than a week means that about 900 individuals can be computed; a typical choice is to retain 36 individuals in the population and to let this population evolve for 24 generations.

\section{Shape Optimization for Airfoils in Transonic Flows of Dense Gases}

\section{A. Drag Minimization for a Symmetric Airfoil}

The problem is to find the minimal value of the drag coefficient $C_{D}(\Sigma)$, where $\Sigma$ denotes an airfoil symmetric shape. In the present case, this shape is constrained to satisfy the following conditions: 1 ) the coordinates of the leading edge and trailing edge normalized by the airfoil chord are, respectively, $(0,0)$ and $(1,0)$ and 2$)$ the thickness-to-chord ratio is $12 \%$. The upper airfoil surface is represented by a Bézier curve that is determined in the present study by the coordinates of eight Bézier points: the fixed leading edge $P_{0}$ and trailing edge $P_{7}$; five control points $P_{k=2,6}\left(x_{k}, y_{k}\right)$, regularly spaced along the chord, with the $x_{k}$ coordinates varying in distinct subintervals of 0 and 1 ; and a control point $P_{1}\left(0, y_{1}\right)$, which ensures that the upper surface of the profile is tangent to the $y$ axis at the leading edge. A fixed $12 \%$ thickness-to-chord ratio is obtained by ad hoc normalization of the airfoil maximum thickness. The family of airfoil shapes considered in the present study is therefore entirely



Fig. 6 Geometry of optimized, sonic arc, Rusak-Wang low-drag, and NACA0012 airfoils.

described by 11 parameters that vary continuously between prescribed limiting values; the shape representation is such that it allows recovering the NACA0012 airfoil and the low-drag airfoil proposed in [16].

The minimization of $C_{D}(\Sigma)$ is first performed for three different values of the freestream Mach number, successively taken equal to $M_{\infty}=0.94,0.95$, and 0.98 . In each case, the flow over a population of evolving (half)-airfoil shapes is computed using the UGS on the grid described in Sec. III.A. The VDW equation of state is used with $\gamma=1.02$, and the freestream thermodynamic conditions previously given in Sec. III.A to allow a comparison with the results obtained in [17]. For each run, the GA-driven population appears to have reached a stabilized optimal (minimal) value of the drag coefficient after the prescribed number of generations, as can be deduced from Fig. $\underline{5}$. The



a)



b)

Fig. 7 Pressure drag vs freestream Mach number for different airfoil shapes; results obtained using a) UGS and b) SGS. 
maximum and mean value of the drag coefficient in the final population of airfoil shapes are almost the same in each case, which means that the algorithm has converged to a population of almost identical geometries. The optimized airfoil shape for each freestream Mach number is plotted in Fig. 6, along with the NACA0012 airfoil, the modified low-drag airfoil, and the sonic arc airfoil derived by Rusak and Wang in [17]. It is noteworthy to observe that the optimal shape for $M_{\infty}=0.94$ (indicated in the following as OA094) turns out to be almost superimposed with the Rusak and Wang low-drag airfoil; consequently, the latter shape will be retained in the following analysis as the shape producing minimal drag at $M_{\infty}=0.94$. Reducing the drag for higher values of the freestream Mach number leads to airfoil shapes that are getting closer to the limiting case of the sonic arc, as far as allowed by the airfoil shape representation retained in this study. Because, ideally, a consistent drag reduction is expected for the whole range of freestream Mach numbers, the wave drag produced by these modified shapes, which are optimized for a specific value of $M_{\infty}$, are next checked for different values of $M_{\infty}$, taken in the interval between 0.9 and 0.995 ; this a posteriori analysis is performed using both the SGS and the UGS for cross-validation purposes. The computed evolutions of the drag coefficient with increasing values of the freestream Mach number are plotted in Fig. 7. The optimal shape at $M_{\infty}=0.95$ (OA095) allows obtaining lower drag levels than the OA094 airfoil for the whole range of freestream Mach numbers, both shapes being far superior to the original NACA0012 airfoil. On the contrary, the optimal shape at $M_{\infty}=0.98$ (OA098) displays a very poor performance for lower values of the freestream Mach number. All of the drag curves fall

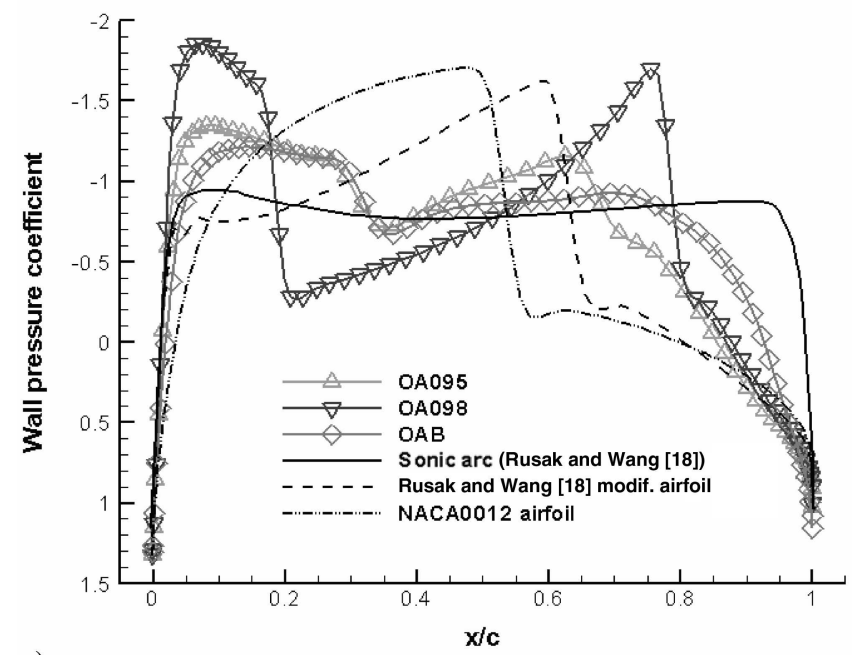

a)

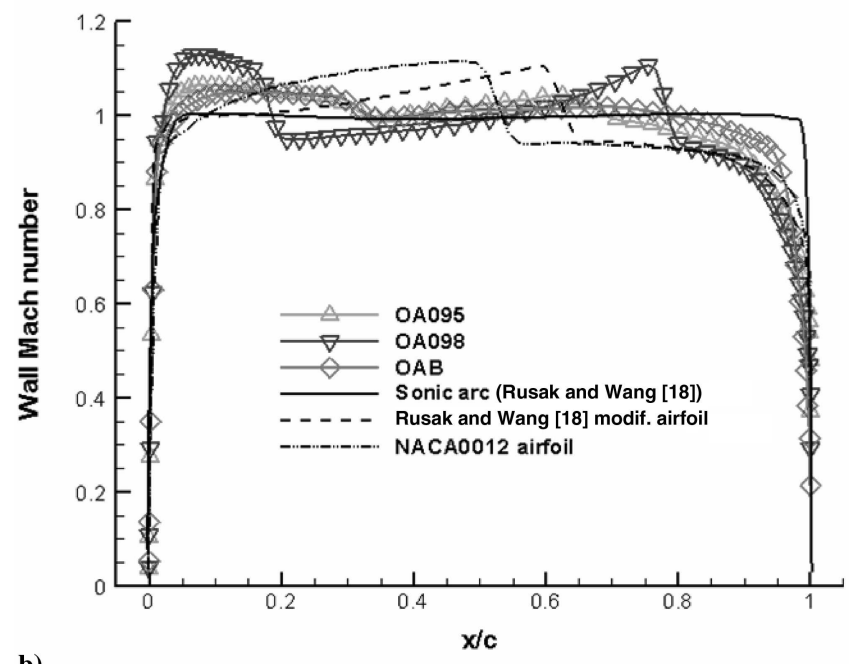

b)

Fig. 8 Distributions at $M_{\infty}=0.95$ for a) wall pressure and b) Mach number; SGS results. within the envelope corresponding to the sonic arc performance, which indeed represents the optimal shape for inviscid flow at transonic speeds and zero angle of attack. The MOGA strategy offers the possibility of looking for an airfoil that would simultaneously minimize the drag coefficient at $M_{\infty}=0.95$ and $M_{\infty}=0.98$. Such an airfoil should provide a good drag performance over a larger range of freestream Mach numbers. To obtain such an airfoil, a bi-objective optimization run was computed using the same GA parameters as in the previous mono-objective runs; among the set of optimal shapes provided by the MOGA, a typical tradeoff solution was retained (denoted as OAB to recall its results from a bi-objective optimization), which is plotted in Fig. 6. The associated drag evolution is displayed in Fig. 7; the OAB airfoil turns to yield lower drag levels than the previous single operation point optima for the whole range of freestream Mach number, because its geometry is actually closer to that of the sonic arc. The wall pressure and Mach number distributions at $M_{\infty}=0.95$ and $M_{\infty}=0.98$ are plotted in Figs. 8 and 9 , respectively, for the various geometries under study. At $M_{\infty}=0.9 \overline{5}$, all of the airfoils, except the sonic arc, are beyond their respective critical Mach number; precisely, the NACA0012, RusakWang, and OA098 airfoils are also beyond the drag divergence Mach number, whereas the OA095 and OAB airfoils still yield almost isentropic flow. Note that the OA098 airfoil displays a shock in the leading-edge region down to $M_{\infty}=0.90$. At $M_{\infty}=0.98$, all of the airfoils, including the sonic arc, are subjected to the transonic drag rise: the OA095 and OAB wall distributions are similar to those associated with the sonic arc up to $70 \%$ of chord, in which the shapes of the three airfoils start to strongly differ (see Fig. 6).

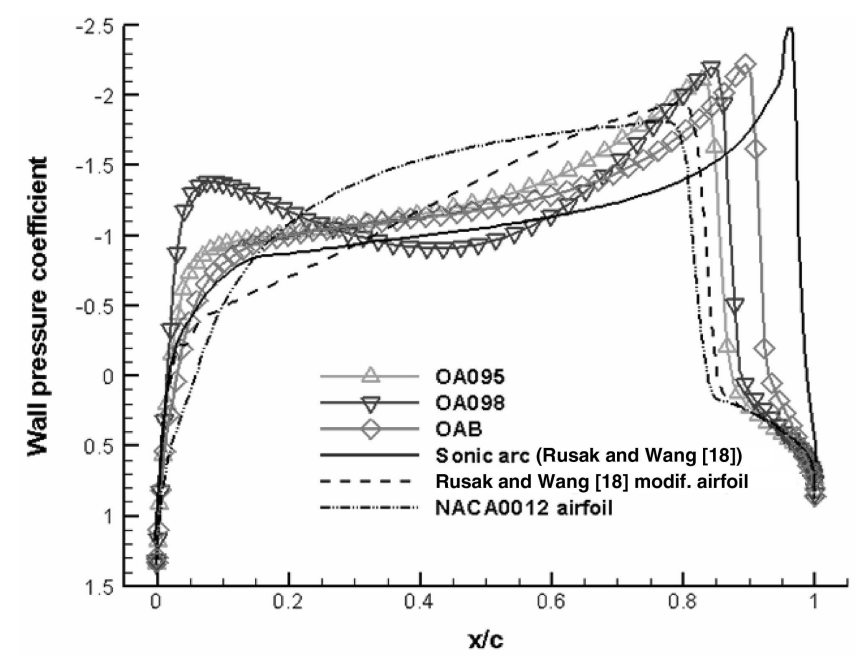

a)

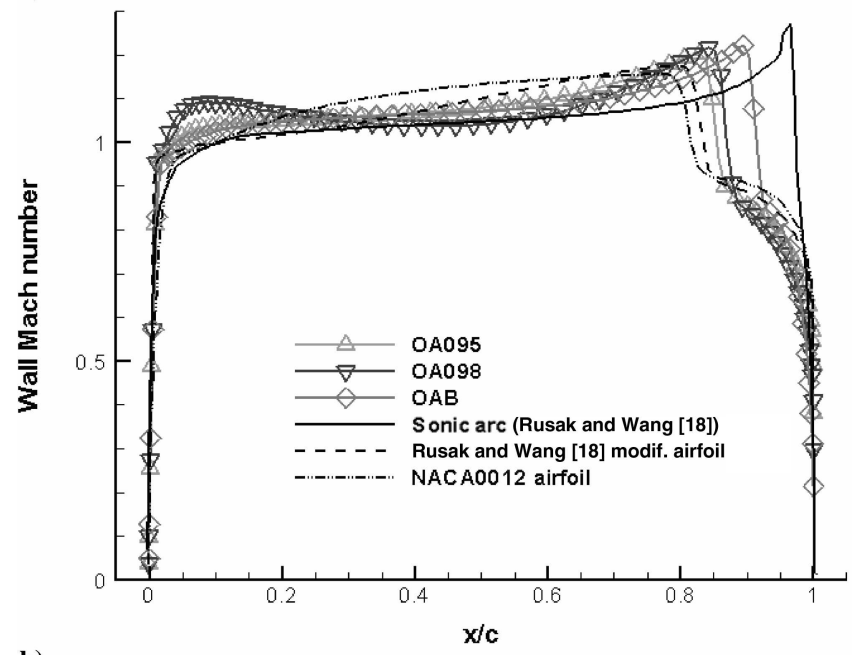

b)

Fig. 9 Distributions at $M_{\infty}=0.98$ for a) wall pressure and b) Mach number distributions; SGS results. 
A similar optimization process was carried out for a flow of perfect gas with $\gamma=1.4$ and for a DG flow with $p_{\infty} / p_{c}=1.0696$ and $\rho_{\infty} / \rho_{c}=0.73502$, modeled using the more realistic MAH equation of state. Although the VDW equation of state was retained up to now to allow a comparison with the results obtained in [17], the analysis performed with MAH and PFG indicates the improvement in terms of drag reduction that can be expected (for inviscid flows) when switching from air to PP10 with airfoil shapes optimized according to the nature of the fluid. Various optimization strategies were applied to the PFG case: single-point drag minimization for different freestream Mach numbers in the transonic drag rise region and simultaneous minimization of drag for two values of $M_{\infty}$ above the drag divergence Mach number of the baseline NACA0012 airfoil. The overall best performance for the shape representation described at the start of this section is plotted in Fig. 10; the optimal airfoil, denoted as OA083, is specifically targeted to achieve minimal (zero) drag at $M_{\infty}=0.83$, and so it suffers from a slight increase in wave drag at $M_{\infty}=0.82$, due to the appearance of a weak shock wave. For the MAH case, the best performance was obtained with an airfoil (OAB) designed to minimize drag at $M_{\infty}=0.925$ and $M_{\infty}=0.95$. As shown in Fig. 10, the optimal airfoil increases the drag divergence number from $M_{\infty}=0.91$ for the NACA0012 airfoil, up to almost $M_{\infty}=0.93$. Figure 11 displays the strong discontinuities produced by the baseline NA $\overline{C A} 0012$ airfoil in the drag divergence region associated with each gas model (PFG, VDW, and MAH) and presents the pressure contours of the corresponding shock-free airfoils (just before the drag divergence Mach number is attained).

As already shown, the optimization procedure leads to minimaldrag airfoils with increasingly better performance as their shape tends to become closer to the sonic arc. Unfortunately, the sonic arc shape is not optimal if the flow of a viscous fluid is considered, because of its blunt trailing edge that can lead to flow separation. For this reason, the Rusak-Wang low-drag airfoil is essentially obtained by modifying the trailing edge of the optimal sonic arc shape, replacing it with a sharp tail similar to that of the NACA0012 airfoil.



a)

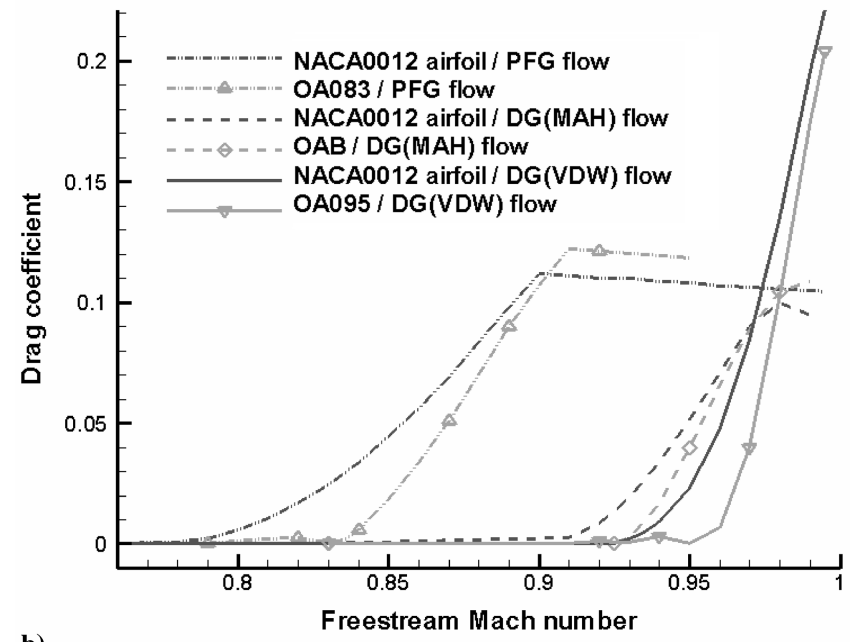

b)

Fig. 10 Effect of the equation of state on optimization results a) optimal geometries for the drag minimization problem solved in the case of a PFG flow and a DG flow with VDW or MAH EOS and b) associated pressure drag as a function of the freestream Mach number.
To check the viscous performance of the optimal shapes derived from the present MOGA optimization procedure, a numerical investigation of viscous turbulent flows past such optimal airfoils is undertaken. In particular, viscous flows past the OA095 and OAB airfoils, which were found to provide the best inviscid performance in the previous study of Rusak's optimization problem, are computed and compared with results obtained for the reference NACA0012 and Rusak-Wang airfoils in the same conditions. The numerical methodology adopted for the study is presented in [15]. The SGS scheme described in Sec. III is extended to viscous flow computations using a centered second-order approximation of the viscous fluxes. Ad hoc thermophysical models [38] are used to describe the variation of the dynamic viscosity and thermal conductivity of dense gases with temperature. Turbulence effects are taken into account using the very simple Baldwin-Lomax model: the underlying working hypothesis is that, to a first approximation, the turbulence structure is not affected by dense-gas effects for thermodynamic conditions sufficiently far from the liquid/vapor critical point. The computations are performed using half-O-grids of $100 \times 64$ cells, with a mean first cell height of about $6 \times 10^{5}$, representing a reasonable tradeoff between accuracy and computational cost. The gas thermodynamic response is modeled using the MAH equation of state. The thermodynamic freestream conditions are taken equal to $p_{\infty} / p_{c}=0.985$ and $\rho_{\infty} / \rho_{c}=0.622$, the Mach number is varied in the interval between 0.85 and 0.99 , and the flow Reynolds number based on freestream conditions and the airfoil chord is $10^{7}$. The flow past the NACA0012 and Rusak-Wang airfoils is attached for freestream values of Mach number less than about 0.97 . In both cases, the flow at $M_{\infty}=0.98$ becomes separated close to the trailing edge, and the flow at $M_{\infty}=0.99$ is massively separated. Conversely, the flowfield around the OAB airfoil already exhibits a recirculation region close to the trailing edge for the lowest values of $M_{\infty}$. In fact, this airfoil shape is the closest to the sonic arc, which greatly enhances its inviscid performance, but represents a serious drawback for viscous flows. An intermediate behavior is found for OA095, because the flow becomes separated at about $M_{\infty}=0.95$ for that shape. Figure 12 shows the flowfields (Mach number contours and streamlines) for the four airfoils at $M_{\infty}=0.96$; the flow past $\mathrm{OAB}$ is characterized by significant separation at the trailing edge, the flow past OA095 is just slightly separated, and the flows past the NACA0012 and Rusak-Wang airfoils are completely attached. Figure 13 shows the drag coefficient vs the Mach number for the four airfoils analyzed. For the lowest values of $M_{\infty}$, the thicker trailing edges of OA095 and OAB lead to poorer performances in comparison with the more slender NACA0012 and Rusak-Wang airfoils. On the other hand, for higher values of $M_{\infty}$, the flow becomes supercritical and wave drag appears; in these conditions, the adapted design of the OA095 and OAB airfoils becomes advantageous, and both airfoils represent a true improvement over the two reference shapes. Globally, the best overall performance over the explored range of Mach numbers is offered by the OA095 airfoil.

\section{B. Performance Optimization for a Lifting Airfoil}

The shape representation retained for the complete airfoil derives from that adopted in the study of a half-profile. The upper part of the airfoil is represented as described in the previous subsection; the lower part is described in a similar way using a Bézier curve based on eight control points, including the fixed leading and trailing edges, as well as a point on the negative part of the $y$ axis that ensures the airfoil leading edge is tangent to the $y$ axis. Once the whole airfoil is generated, the thickness distribution is normalized to yield a $12 \%$ thickness-to-chord ratio.

The lift coefficient in the OP 1 conditions, $C_{L}(\Sigma ;$ OP 1$)$, and the lift-to-drag ratio in the OP 2 conditions, $\left(C_{L} / C_{D}\right)(\Sigma$; OP 2$)$, are simultaneously maximized by applying the MOGA with a population of 36 individuals during 24 generations. A partial view of the computed individuals is shown in Fig. 14, along with the set of nondominated solutions eventually obtained. Figure 14 clearly illustrates that the initial NACA0012 airfoil aerodynamic perform- 
ances in DG flow were substantially improved through the optimization process. In particular, almost all of the Pareto-optimal individuals now have a lift coefficient at OP 1 and a lift-to-drag ratio at OP 2 greater than the lift coefficient and the lift-to-drag ratio generated by the NACA0012 airfoil for a DG flow. To draw a fair comparison with PFG flow, a shape optimization is also performed for the perfect-gas $(\gamma=1.4)$ flow at $M_{\infty}=0.85$ and $1 \mathrm{deg}$ of incidence over an airfoil of $12 \%$ thickness-to-chord ratio, with a geometry based on the Bézier curves previously described. With unique thermodynamic conditions for this PFG flow, the problem is expressed as the simultaneous maximization of the lift and minimization of the drag. The optimal solutions obtained are plotted in Fig. 14 in the lift/lift-to-drag plane: they remain well below the optimal solutions associated with the DG flow. Figure 15 displays one of the optimal solutions selected along the final Pareto front; the $\mathrm{OAB}$ shape was retained because, among the set of nondominated solutions, it displays the thickness distribution in the trailing-edge region that is the most likely to preserve good aerodynamic performances in the viscous case. The wall pressure and Mach number distributions computed on the NACA0012 airfoil and OAB for subcritical (OP 1) and supercritical (OP 2) conditions are plotted in Figs. 16 and 17. For subcritical conditions, both airfoils display zero drag, but $\mathrm{OAB}$ yields a much higher lift coefficient $\left(C_{L}=0.794\right)$ than the NACA0012 airfoil $\left(C_{L}=0.226\right)$, due to a much stronger expansion of the flow around the upper leading edge. For supercritical conditions, the $\mathrm{OAB}$ yields more than twice the drag of the NACA0012 airfoil $\left(C_{D}=0.116\right.$ vs $\left.C_{D}=0.051\right)$, but also generates almost three times the lift of the NACA0012 airfoil $\left(C_{L}=1.3\right.$ vs $\left.C_{L}=0.45\right)$, and so its lift-to-drag ratio of 11.2 is indeed better than the value of 8.8 obtained with the NACA0012 airfoil, as expected from the optimization process. An overview of the optimized airfoil performance is provided in Fig. 18, in which the lift coefficient and the lift-to-drag ratio are plotted for dense-gas flow around both the baseline NACA002 airfoil and the optimized airfoil for different freestream thermodynamic conditions covering the subcritical as well as the low- and high-pressure transonic BZT flow regimes. As expected from the two-point optimization, the modified airfoil systematically ensures a level of lift for DG flow in the subcritical regime that is at least equivalent to the highest level achievable with a PFG flow, while preserving a high lift-to-drag ratio in the subcritical and low-pressure supercritical regimes.

The airfoil viscous performance has also been checked for this lifting case, using the methodology of [15]. The viscous computations were performed on an O-grid of $200 \times 64$ cells, with a mean first cell height in the direction normal to the wall equal to about $6 \times 10^{-5} c$, and on a finer grid of $200 \times 128$ cells, with a mean first cell height equal to $5 \times 10^{-6} c$. The outer boundary is located about 12 chords away from the airfoil. The freestream conditions are successively taken equal to OP 1 and OP 2 . The corresponding finegrid values of the lift and drag coefficients (respectively, $\mathrm{C}_{L}$ and $\mathrm{C}_{D}$ ) and of the lift-to-drag ratio are reported in Table 1 . For comparison, viscous solutions for DG flows past the NACA 0012 airfoil at the same thermodynamic conditions are also presented. The optimized airfoil conserves a superior aerodynamic performance over the
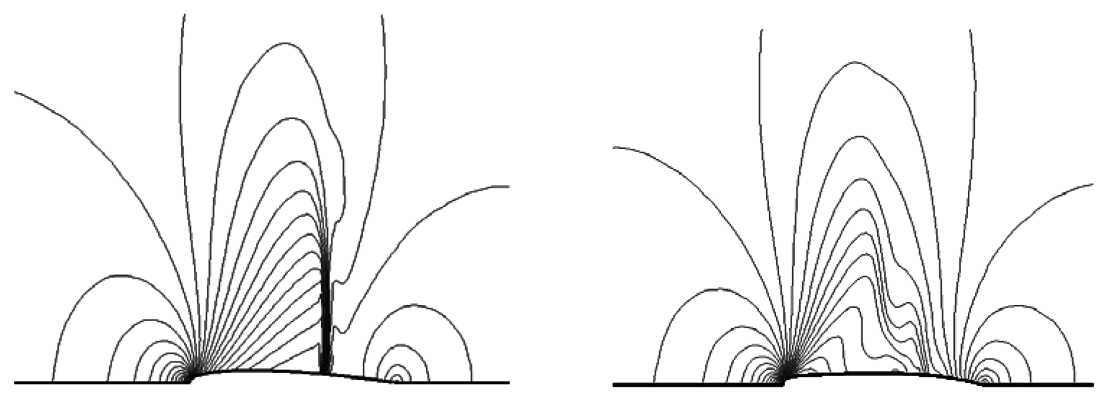

a) Perfect-gas flow: $M_{\infty}=\mathbf{8 3 . 0}$


b) Dense-gas flow, VDW equation of state: $M_{\infty}=0.95$


c) Dense-gas flow, MAH equation of state: $M_{\infty}=0.925$

Fig. 11 Pressure contours around the baseline NACA0012 airfoil and optimal airfoils for perfect and dense-gas flows with freestream conditions leading to an equivalent amount of wave drag for the baseline airfoil. 


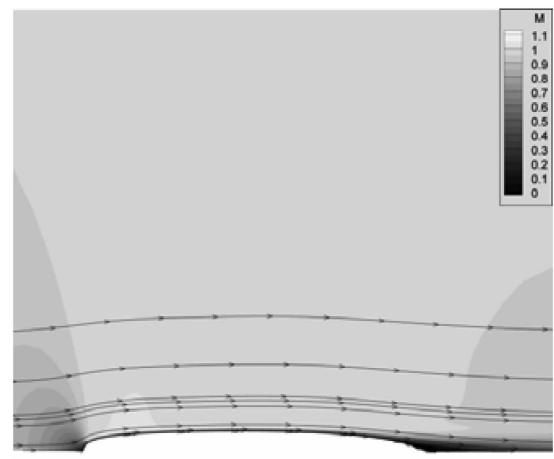

a)

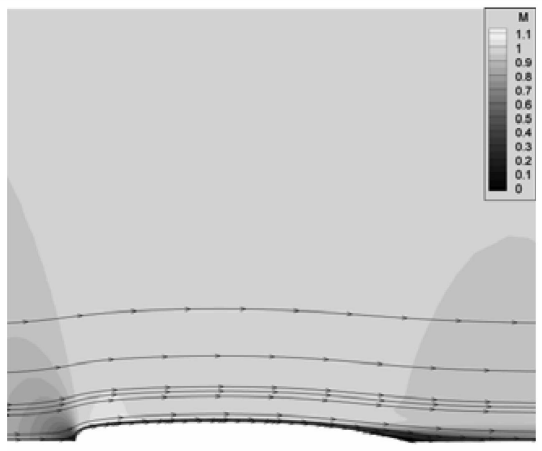

b)

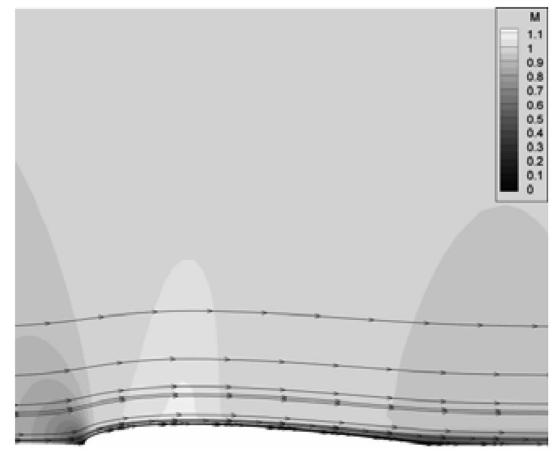

c)



d)
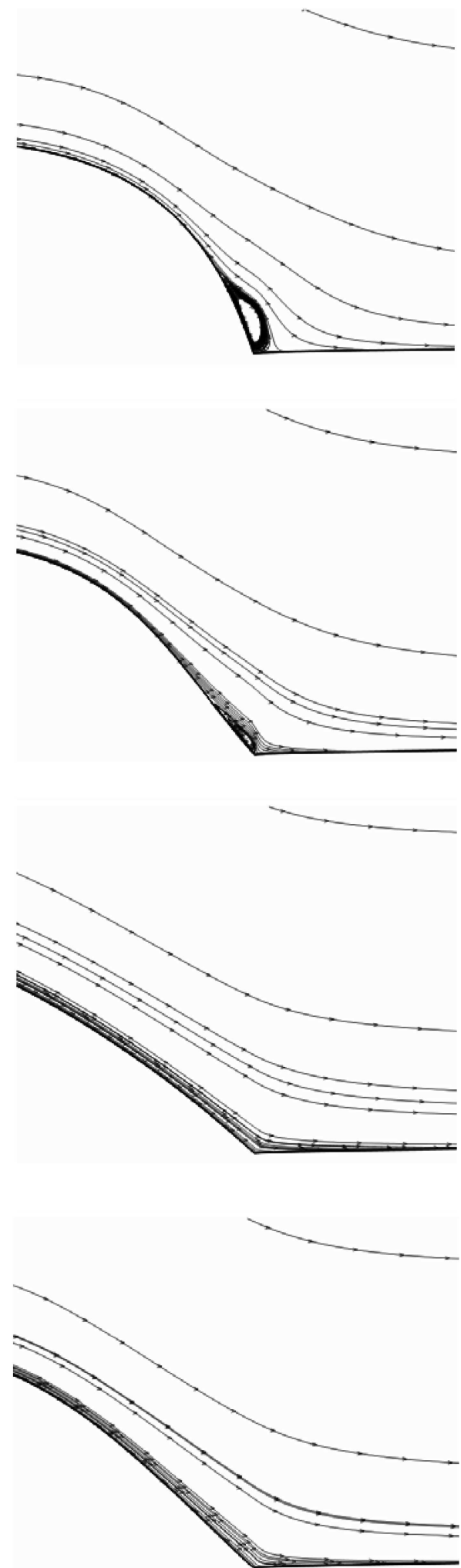

Fig. 12 Mach number contours and streamlines for turbulent flows past four airfoils and close-up of the streamlines at the trailing edge (grid aspect ratio not preserved): a) $\mathrm{OAB}$, b) OA095, c) NACA0012, and d) Rusak-Wang; $M_{\infty}=0.96$ and $R e \cong 10^{7}$.

NACA0012, even when viscous effects are relevant. It should be noticed, however, that the viscous performance of both DG and PFG flows significantly differs from inviscid results. At flow conditions OP 1 , the flowfield is entirely subsonic and the boundary layer is attached, both for the NACA0012 and for the OAB airfoils. However, because of the high total pressure of the incoming stream, the flow strongly accelerates downstream of the stagnation point, and a deep suction peak appears at the upper surface downstream of the leading edge. In fact, the strong suction exerted on the upper surface is the main reason for the dramatic improvement of the lift over the perfect gas for an inviscid flow. However, when viscous effects are taken into account, the very strong adverse pressure gradient downstream of the suction peak leads to a significant growth of the boundary layer and to enhanced friction in the leading-edge region. Consequently, the peak itself is noticeably smoothed out, and the airfoil lift drops below the inviscid value. Figure 19 shows the wall 


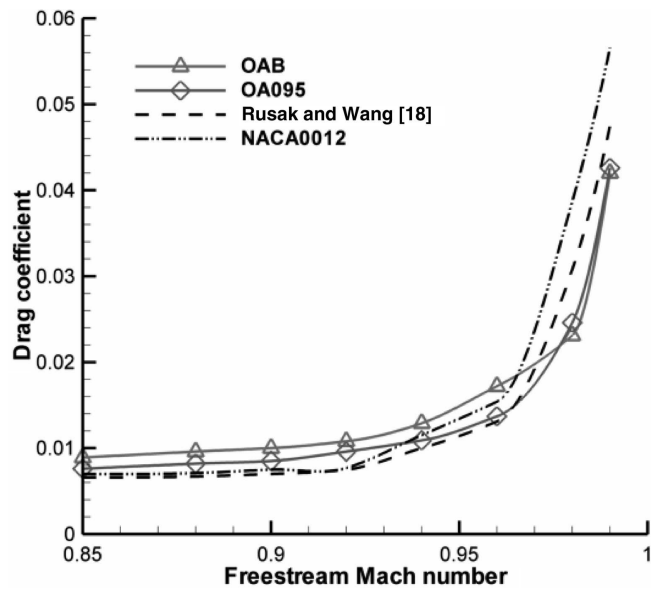

Fig. 13 Viscous performance of minimal-drag airfoils stemming from inviscid optimization.

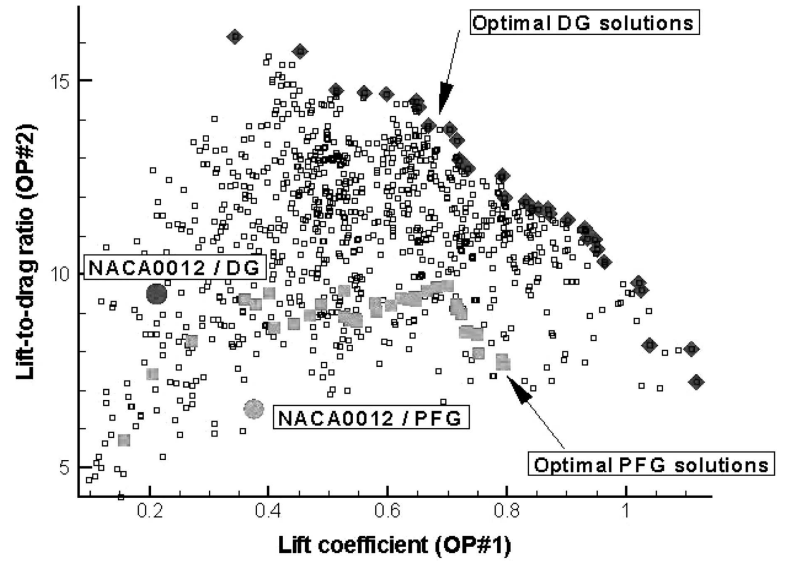

Fig. 14 Lifting-airfoil two-point performance optimization; overview of the computed solutions during the genetic evolution process.

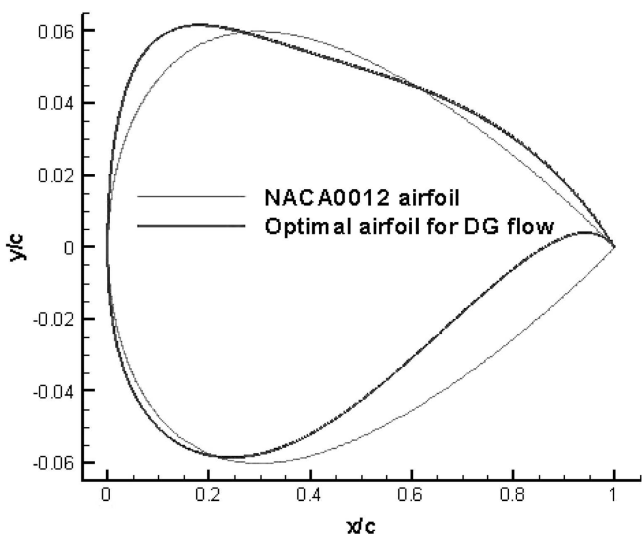

Fig. 15 Lifting-airfoil two-point performance optimization; typical optimal shape.

pressure coefficient and skin friction distribution for this case. For flow condition OP 2, the relevant differences with respect to inviscid flow are due to strong shock/boundary-layer interactions, leading to postshock separation and reattachment, both in the flow over the NACA0012, and over OAB, with subsequent blowup of the subsonic part of the boundary layer (see the wall pressure and skin friction distributions in Fig. 20). Paradoxically, in this case, the shock/ boundary-layer interaction leads to a reduction in both lift and drag coefficients with respect to inviscid flow, due to the considerably


Fig. 16 Lifting-airfoil two-point performance optimization; wall distributions at OP 1 for the NACA0012 airfoil and an optimal airfoil for DG flow.

decreased strength of the shock waves. For completeness, the PFG viscous aerodynamic performance of the NACA0012 is also included in Table 1. In the PFG case, the lift coefficient is negative, because of massive postshock separation at the upper surface, caused by severe shock/boundary-layer interaction (see [14]).
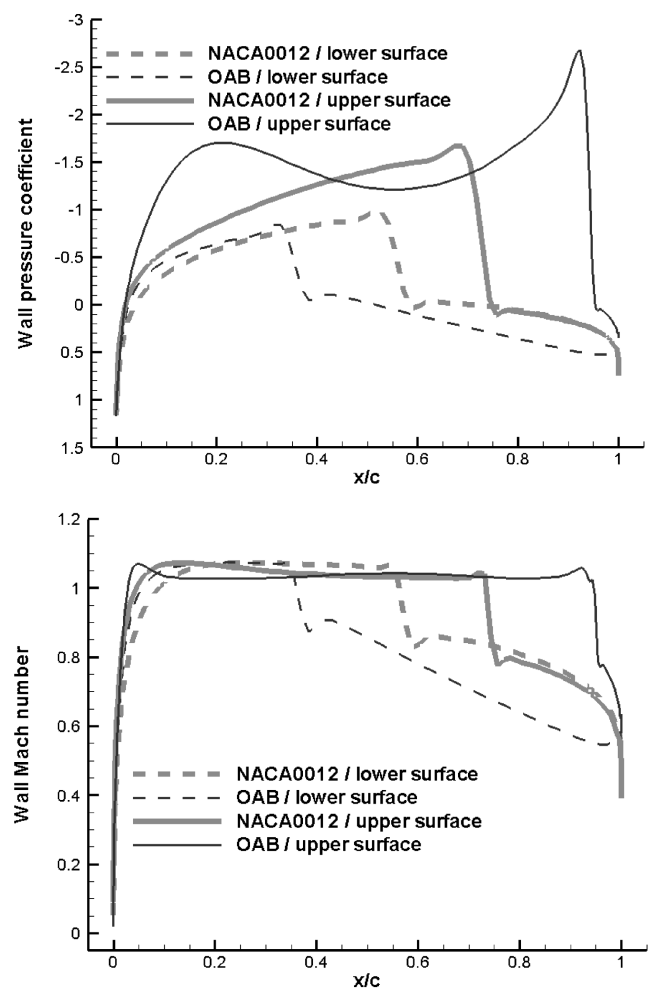

Fig. 17 Lifting-airfoil two-point performance optimization; wall distributions at OP 2 for the NACA0012 airfoil and an optimal airfoil for DG flow. 



Fig. 18 Evolution of aerodynamic performances at $M_{\infty}=0.85$ and $\alpha=1 \mathrm{deg}$ for a dense-gas flow around the NACA0012 airfoil and a DGoptimized airfoil at different freestream thermodynamic conditions.

\section{Conclusions}

A shape optimization was performed for inviscid transonic flows of dense gases over airfoils. Though computationally intensive, the use of a genetic algorithm to solve this optimization has the following advantages:

1) Because the GA is fully decoupled from the solver providing the values of the objective functions to be optimized, it allows an easy switch from the simple Van der Waals equation of state to the more advanced Martin-Hou equation of state.

2) A GA is well adapted to the solution of multipoint optimization problems that arise naturally in aerodynamics (simultaneous drag minimization for near-critical and supercritical Mach numbers or performance improvement for a lifting airfoil in both the subcritical and supercritical regimes).

When solved for inviscid flow, the drag minimization problem of a symmetric airfoil with fixed thickness-to-chord ratio indeed yields shock-free shapes for an extended range of freestream Mach number. However, the optimal airfoils display a thick trailing edge that is bound to induce premature flow separation; hence, an increase in form drag when taking into account the viscous effects in the flow analysis. This point was actually checked by a posteriori numerical experiments using an available version of the SGS solver extended to the Reynolds-Averaged Navier-Stokes (RANS) equations for dense-gas flows. The multipoint performance optimization for a lifting airfoil in subcritical and supercritical flow conditions allowed determining an airfoil shape that provides an overall improvement of the lift coefficient while preserving the high lift-to-drag ratio typical from BZT flows. However, in the case of inviscid flow optimization, it was again necessary to select an optimum shape within the optimal Pareto set that might also ensure good performances for viscous flows, thanks to a thinner trailing edge, less prone to boundary-layer separation. Further developments of the study will aim at taking into
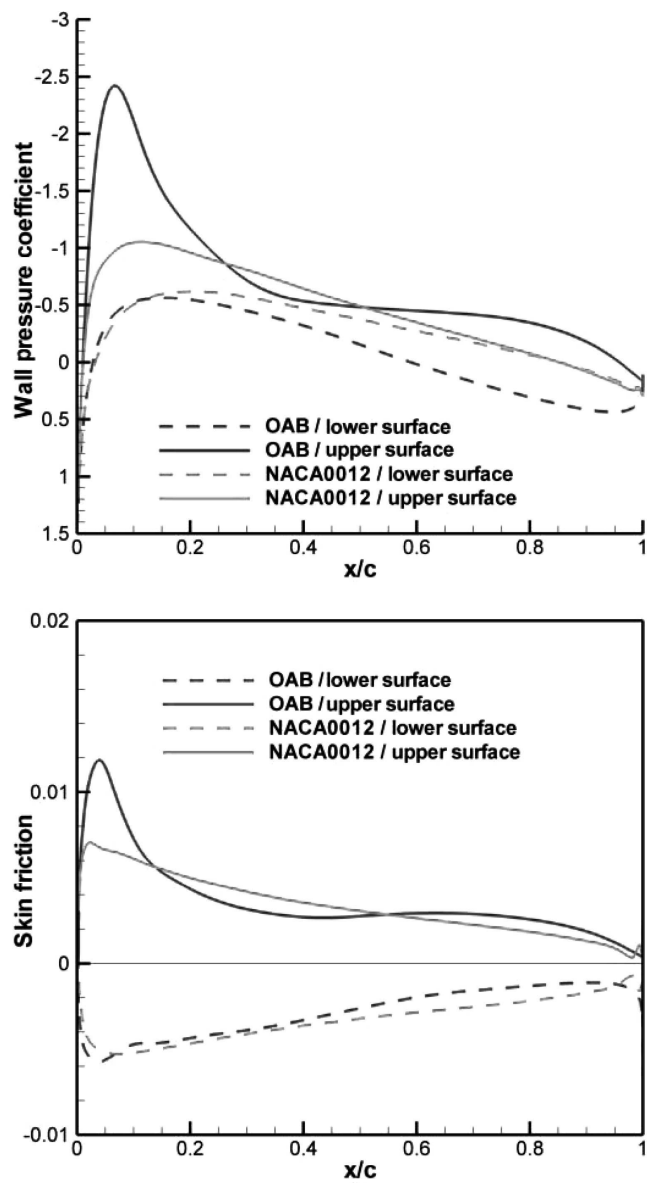

Fig. 19 Lifting-airfoil two-point performance optimization; wall distributions of the pressure and skin friction coefficients for viscous flow at OP 1 past the NACA0012 airfoil and an optimal airfoil for DG flow.

account viscous effects directly within the optimization process, rather than by an a posteriori analysis. A substantial reduction of computational costs is necessary to carry out such a RANS-based optimization, which can be achieved by reducing the number of evaluations of the objective function via the Navier-Stokes solver by the use of properly calibrated artificial neural networks [33]; on the other hand, genetic algorithms are naturally well adapted to parallel computing. Moreover, significant improvement may be expected from the use of hybrid optimization algorithms, combining the flexibility of genetic strategies with the accuracy and efficiency of gradient-based methods. As a final remark, note that the isolated airfoils considered in the present study merely represent a useful simplified configuration to perform preliminary feasibility studies. The actual interest lies instead in the application of the present results to ORC turbine cascades. Preliminary studies about inviscid and viscous dense-gas flows through turbine cascades $[39,40]$ have shown that the use of a BZT working fluid allows an efficiency improvement of about $3 \%$ over air, and even greater benefits with

Table 1 Aerodynamic performance of viscous flows over the NACA0012 airfoil and an optimized airfoil stemming from the MOGA strategy

\begin{tabular}{lccc}
\hline \hline Case & $C_{D}$ & $C_{L}$ & $C_{L} / C_{D}$ \\
\hline $\begin{array}{l}\text { Optimized airfoil, } \\
\text { condition OP 1 }\end{array}$ & $1.416 \times 10^{-2}$ & 0.6077 & 42.92 \\
$\begin{array}{l}\text { Optimized airfoil, } \\
\quad \text { condition OP 2 }\end{array}$ & $6.648 \times 10^{-2}$ & 0.9693 & 14.58 \\
NACA0012, condition OP 1 & $1.275 \times 10^{-2}$ & 0.1833 & 14.38 \\
NACA0012, condition OP 2 & $3.448 \times 10^{-2}$ & 0.2529 & 7.335 \\
NACA0012, perfect-gas flow & $5.456 \times 10^{-2}$ & $-4.086 \times 10^{-2}$ & -0.7489 \\
\hline \hline
\end{tabular}



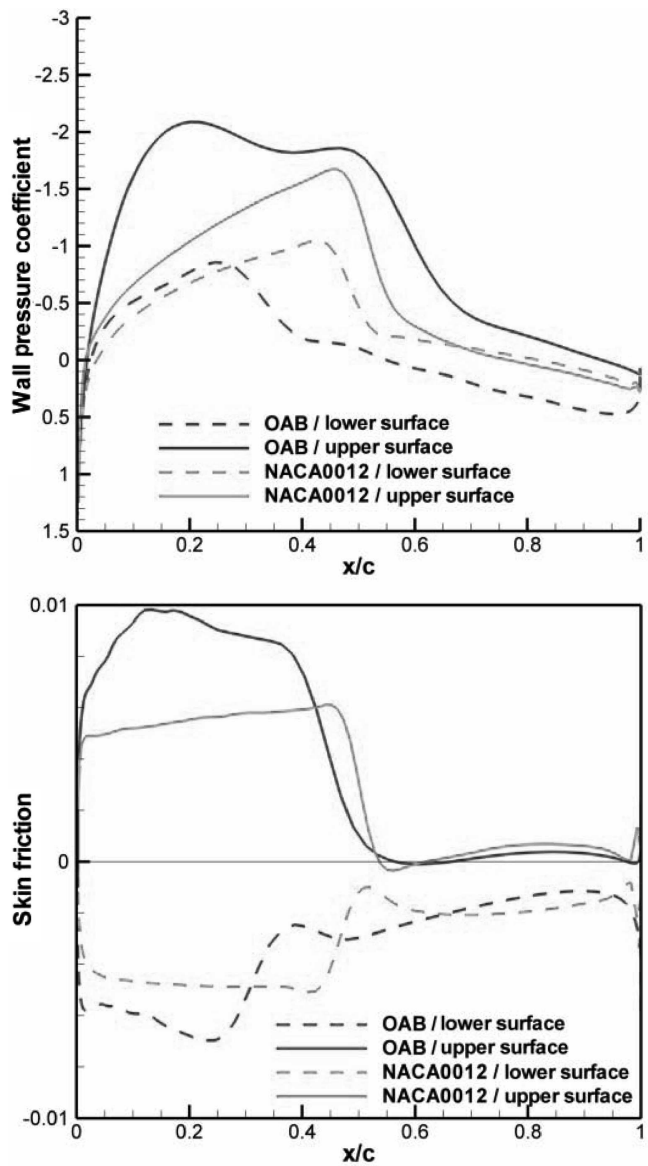

Fig. 20 Lifting-airfoil two-point performance optimization; wall distributions of the pressure and skin friction coefficients for viscous flow at OP 2 past the NACA0012 airfoil and an optimal airfoil for DG flow.

respect to steam, for a given cascade pressure ratio. The preceding advantages were simply due to the special nature of the working fluids, because the blade shapes considered for the study were typical gas turbine blade sections, not specifically adapted for dense-gas flows. The present encouraging results make one reasonably believe that a proper multipoint shape optimization could further improve the system efficiency over a sufficiently wide range of operating conditions, while conserving the same cascade pressure ratio of the baseline flow, that is, without significantly affecting the system power output. This would be a fundamental breakthrough for the development of real-world BZT ORC turbogenerators.

\section{References}

[1] Thompson, P. A., "A Fundamental Derivative in Gas Dynamics," Physics of Fluids, Vol. 14, No. 9, 1971, pp. 1843-1849.

[2] Cramer, M. S., and Kluwick, A., "On the Propagation of Waves Exhibiting Both Positive and Negative Nonlinearity," Journal of Fluid Mechanics, Vol. 142, May 1984, pp. 9-37.

[3] Cramer, M. S., "Shock Splitting in Single-Phase Gases," Journal of Fluid Mechanics, Vol. 199, 1989, pp. 281-296.

[4] Cramer, M. S., and Best, L. M., "Steady, Isentropic flows of Dense Gases in Cascade Configurations," Physics of Fluids A, Vol. 3, No. 1, 1991, pp. 219-226.

[5] Cramer, M. S., and Tarkenton, G. M., "Transonic Flows of BetheZel'dovich-Thompson Fluids," Journal of Fluid Mechanics, Vol. 240, July 1992, pp. 197-228.

[6] Monaco, J. F., Cramer, M. S., and Watson, L. T., "Supersonic Flows of Dense Gases in Cascade Configurations," Journal of Fluid Mechanics, Vol. 330, Jan. 1997, pp. 31-59.

[7] Brown, B. P., and Argrow, B. M., "Application of Bethe-Zel'dovichThompson Fluids in Organic Rankine Cycles," Journal of Propulsion and Power, Vol. 16, No. 6, 2000, pp. 1118-1124.

[8] Lambrakis, K. C., and Thompson, P. A., "Existence of Real Fluids with a Negative Fundamental Derivative $\Gamma$," Physics of Fluids, Vol. 15 , No. 5, 1972, pp. 933-935.

[9] Thompson, P. A., and Lambrakis, K. C., "Negative Shock Waves," Journal of Fluid Mechanics, Vol. 60, Aug. 1973, pp. 187-208.

[10] Cramer, M. S., "Negative Nonlinearity in Selected Fluorocarbons," Physics of Fluids A, Vol. 1, No. 11, 1989, pp. 1894-1897.

[11] Cinnella, P., and Congedo, P. M., "Aerodynamic Performance of Transonic Bethe-Zel'Dovich-Thompson Flows Past an Airfoil," AIAA Journal, Vol. 43, No. 2, 2005, pp. 370-378.

[12] Cinnella, P., Congedo, P. M., and Laforgia, D., "Investigation of BZT Transonic Flows Past an Airfoil Using a 5th Power Virial Equation of State," Proceedings of the 4th European Congress on Computational Methods in Applied Sciences and Engineering [CD-ROM], Vol. 1, Univ. of Jyvaskyla, Jyvaskyla, Finland, 2004.

[13] Cinnella, P., and Congedo, P. M., "Inviscid and Viscous Behavior of Dense Gas Flows Past an Airfoil" (to be published).

[14] Cinnella, P., "Viscous Performance of Transonic Dense Gas Flows," 35th AIAA Fluid Dynamics Conference and Exhibit, Toronto, Canada, AIAA Paper 2005-5284, 2005.

[15] Cinnella, P., and Congedo, P. M., "Numerical Solver for Dense Gas flows," AIAA Journal, Vol. 43, No. 11, 2005, pp. 2458-2461.

[16] Wang, C. W., and Rusak, Z., "Similarity Solutions of $\phi_{X}^{3} \phi_{X X}=\phi_{\tilde{Y} \tilde{Y}}$ with Applications to Transonic Aerodynamics of Dense Gases," SIAM Journal on Applied Mathematics, Vol. 59, No. 2, 1998, pp. 514-528.

[17] Rusak, Z., and Wang, C. W., "Low-Drag Airfoils in Transonic Flows of Dense Gases," Zeitschrift für angewandte Mathematik und Physik (ZAMP), Vol. 51, No. 3, 2000, pp. 467-480.

[18] Argrow, B. M., "Computational Analysis of Dense Gas Shock Tube Flow," Shock Waves, Vol. 6, No. 4, 1996, pp. 241-248.

[19] Brown, B. P., and Argrow, B. M., "Nonclassical Dense Gas Flows for Simple Geometries," AIAA Journal, Vol. 36, No. 10, 1998, pp. 1842 1847.

[20] Wang, C. W., and Rusak, Z., "Numerical Studies of Transonic BZT gas Flows Around a Thin Airfoil," Journal of Fluid Mechanics, Vol. 396, Oct. 1999, pp. 109-141.

[21] Martin, J. J., and Hou, Y. C., "Development of an Equation of State for Gases," AIChE Journal, Vol. 1, No. 2, 1955, pp. 142-151.

[22] Emanuel, G., "Assessment of the Martin-Hou Equation for Modelling a Nonclassical Fluid," Journal of Fluids Engineering, Vol. 116, No. 4, 1994, pp. 883-884.

[23] Guardone, A., Vigevano, L., and Argrow, B. A., "Assessment of Thermodynamic Models for Dense Gas Dynamics," Physics of Fluids, Vol. 16, No. 1, 2004, pp. 3878-3887.

[24] Jameson, A., Schmidt, W., and Turkel, E., "Solutions of the Euler Equations by Finite Volume Methods Using Runge-Kutta TimeStepping Schemes," AIAA Paper 81-1259, June 1981.

[25] Rezgui, A., Cinnella, P., and Lerat, A., "Third-Order Finite Volume Schemes for Euler Computations on Curvilinear Meshes," Computers and Fluids, Vol. 30, Nos. 7-8, 2001, pp. 875-901.

[26] Harten, A., Lax, P. D., and van Leer, B., "On Upstream Differencing and Godunov-Type Schemes for Hyperbolic Conservation Laws," SIAM Review, Vol. 25, No. 1, 1983, pp. 35-61.

[27] Van Leer, B., "toward the Ultimate Conservative Difference Scheme, 5: A Second-Order Sequel to Godunov's Method," Journal of Computational Physics, Vol. 32, No. 2, 1979, pp. 101-136.

[28] Barth, T. J., and Jespersen, D. C., "The Design and Application of Upwind Schemes on Unstructured Meshes," AIAA Paper 89-0366, 1989.

[29] Venkatakrishnan, V., "Convergence to Steady-State Solutions of the Euler Equations on Unstructured Grids with Limiters," Journal of Computational Physics, Vol. 118, No. 1, 1995, pp. 120-130.

[30] Luo, H., Baum, J., and Löhner, R., "A Fast, Matrix-Free Implicit Method for Compressible Flows on Unstructured Grids," Journal of Computational Physics, Vol. 146, No. 2, 1998, pp. 664-690.

[31] Rusak, Z., and Wang, C. W., "Transonic Flows of Dense Gases Around an Airfoil with a Parabolic Nose," Journal of Fluid Mechanics, Vol. 346, Jan. 1997, pp. 1-21.

[32] Wang, J. F., Periaux, J., and Sefrioui, M., "Parallel Evolutionary Algorithms for Optimization Problems in Aerospace Engineering," Journal of Computational and Applied Mathematics, Vol. 149, No. 1, 2002, pp. 155-169.

[33] Giannakoglou, K. C., "Design of Optimal Aerodynamic Shapes Using Stochastic Optimization Methods and Computational Intelligence," Progress in Aerospace Sciences, Vol. 38, No. 1, 2002, pp. 43-76.

[34] Peigin, S., and Epstein, B., "Robust Optimization of 2D Airfoils Driven by Full Navier-Stokes Computations," Computers and Fluids, Vol. 33, No. 9, 2004, pp. 1175-1200.

[35] Pulliam, T. H., Nemec, N., Holst, T., and Zingg, D. W., "Comparison of Evolutionary (Genetic) Algorithm and Adjoint Methods for Multi- 
Objective Viscous Airfoil Optimization," 41st Aerospace Sciences Meeting, Reno, NV, AIAA Paper 2003-0298, 2003.

[36] Deb, K., Multi-Objective Optimization Using Evolutionary Algorithms, Wiley, New York, 2001.

[37] Srinivas, N., and Deb, K., "Multiobjective Function Optimization Using Nondominated Sorting Genetic Algorithms," Evolutionary Computation, Vol. 2, No. 3, 1995, pp. 221-248.

[38] Chung, T. H., Ajlan, M., Lee, L. L., and Starling, K. E., "Generalized Multiparameter Correlation of Nonpolar and Polar Fluid Transport Properties," Industrial and Engineering Chemistry Research, Vol. 27, No. 4, 1988, pp. 671-679.
[39] Cinnella, P., Congedo, P. M., and Laforgia, D., "Transonic Flows of BZT Fluids Through Turbine Cascades," Computational Fluid Dynamics 2004, edited by D. Zingg, Springer-Verlag, Berlin, 2004, pp. 227-232

[40] Cinnella, P., "Numerical Simulations of Dense Gas Flows in Turbomachinery," Proceedings of the TCN-CAE Conference 2005 [CD-ROM], Paper 68, Consorzio TCN, Pula, Italy, 2005.

H. Chelliah Associate Editor 